\title{
Exemestane and Everolimus combination treatment of hormone receptor positive, HER2 negative metastatic breast cancer: A retrospective study of 9 cancer centers in the Campania Region (Southern Italy) focused on activity, efficacy and safety
}

\author{
FERDINANDO RICCARDI $^{1}$, GIUSEPPE COLANTUONI ${ }^{2}$, ANNA DIANA $^{3}$, \\ CARMELA MOCERINO ${ }^{1}$, GIACOMO CARTENİ ${ }^{1}$, ROSSELLA LAURIA ${ }^{4}$, ANTONIO FEBBRARO $^{5}$, \\ FRANCESCO NUZZO $^{6}$, RAFFAELE ADDEO ${ }^{7}$, OMBRETTA MARANO ${ }^{8}$, PASQUALE INCORONATO ${ }^{9}$, \\ SABINO DE PLACIDO ${ }^{4}$, FORTUNATO CIARDIELLO ${ }^{3}$ and MICHELE ORDITURA ${ }^{3}$ \\ ${ }^{1}$ Breast Unit, A. Cardarelli Hospital, I-80131 Naples; ${ }^{2}$ Medical Oncology, San Giuseppe Moscati Hospital, \\ I-83100 Avellino; ${ }^{3}$ Division of Medical Oncology, Department of Precision Medicine, \\ University of Campania 'Luigi Vanvitelli'; ${ }^{4}$ Medical Oncology, Department of Clinical Medicine and Surgery, \\ University of Naples Federico II, I-80131 Naples; ${ }^{5}$ Medical Oncology, Sacro Cuore di Gesù Fatebenefratelli Hospital, \\ I-82100 Benevento; ${ }^{6}$ Breast Cancer Unit, Cancer Institute G. Pascale Foundation, I-80131 Naples; \\ ${ }^{7}$ Medical Oncology, Frattamaggiore Hospital, I-80027 Frattamaggiore; ${ }^{8}$ Medical Oncology, Santa Maria della Pietà Hospital, \\ I-80035 Nola; ${ }^{9}$ Medical Oncology, ASL Napoli 2 Nord Hospital, I-80014 Giugliano, Italy
}

Received January 29, 2018; Accepted June 5, 2018

DOI: $10.3892 / \mathrm{mco} .2018 .1672$

\begin{abstract}
Exemestane (Exe) in combination with Everolimus (Eve) represents an important treatment option for patients diagnosed with hormone receptor positive $(\mathrm{HR}+)$, human epidermal growth factor receptor 2 negative (HER2-) metastatic breast cancer (MBC), which was previously treated with non-steroidal aromatase inhibitors (NSAI). Data from unselected populations may be useful for defining the optimal therapeutic algorithm within a clinical setting. Data from $264 \mathrm{HR}+$, HER2- MBC patients who received Exe-Eve treatment in combination, following the failure of NSAIs was retrospectively analyzed. Different lines of endocrine treatment (ET) were investigated to evaluate the efficacy and toxicity of the treatment within the 'everyday clinical practice' population. The disease control rate (DCR) was $73.1 \%$, with no statistically significant difference among the different settings. At a median follow-up of 42 months, the median progression free survival (PFS) was 11.6, 9.7 and 7.5 months for patients treated with Exe-Eve as first, second or third line therapy, respectively. There was a statistically significant correlation
\end{abstract}

Correspondence to: Dr Anna Diana, Division of Medical Oncology, Department of Precision Medicine, University of Campania 'Luigi Vanvitelli', 5 Sergio Pansini Street, I-80131 Naples, Italy

E-mail: annadiana88@gmail.com

Key words: endocrine therapy, everolimus, metastatic breast cancer, real-life experience with younger age, no previous adjuvant chemotherapy (CT), no previous adjuvant endocrine therapy (ET), HT duration $\geq 36$ months, involvement of liver and/or lung, no prior CT for metastatic disease and $\mathrm{PS}=0$ at the start of treatment. The median overall survival (OS) was 33.0 months; at a median follow-up of 67 months, the median OS was 43.1, 31.7 and 27.9 months in patients treated with Exe-Eve in first, second or third line therapy, respectively. On multivariate analysis, diabetes and previous CT for metastatic disease were revealed to correlate with a worse outcome. Conversely, the presence of mucositis was significantly associated with long-term survival. Overall, Exe-Eve was typically well tolerated and the majority toxicities were $\mathrm{G} 1$ or 2 , while treatment discontinuation due to unacceptable toxicity was only required in $5.7 \%$ of patients. Despite the limitations due to the observational nature of this study, the findings suggest that treatment with Exe-Eve is an active and safe therapeutic option for endocrine-sensitive MBC patients in a real-world clinical setting, regardless of treatment lines.

\section{Introduction}

Breast cancer (BC) is the most common malignancy and the leading cause of cancer death in women worldwide. Approximately $75 \%$ of BCs are hormone receptor positive (HR+), HER2-negative (HER2-); systemic therapy with endocrine agents represents the mainstay of treatment both in the early and advanced stages of disease $(1,2)$.

Considering the efficacy and the favorable safety profile of endocrine-directed agents, sequential lines of endocrine therapy (ET) should be the preferred treatment strategy 
in the advanced setting, except in the case of immediate life-threatening disease or rapid visceral recurrence during adjuvant ET $(3,4)$.

Despite the effectiveness of ET in HR+ advanced BC, disease progression occurs in the majority of patients due to primary or acquired ET resistance.

In the last two decades, loss of estrogen receptor (ER) expression, ER mutations, alterations in co-regulatory proteins, and the upregulation of different signal transduction pathways have been identified as mechanisms leading to ET failure (5).

Among these mechanisms of resistance, cross-talk between the phosphatidylinositol 3-kinase (PI3K)/protein kinase B (AKT)/mTOR axis and ER signaling plays a key role in $\mathrm{BC}$ proliferation and progression and confers endocrine insensitivity to ET.

Preclinical and clinical studies have shown that co-targeting downstream elements of this pathway using mammalian target of rapamycin (mTOR) inhibitors may synergistically increase the antitumoral activity of ET and overcome anti-hormone therapy resistance in $\mathrm{BCs}$ (6-8).

Everolimus (Eve) is a mTOR inhibitor which induces apoptosis by blocking S6K1 and 4E-BP1 activation and inhibits cell growth, proliferation, and G1-S transition. Eve in combination with Exemestane (Exe), a steroidal aromatase inhibitor (SAI), was approved in 2012 based on the results of the pivotal BOLERO-2 trial. In this study, dual-blockade significantly improved progression-free survival (PFS), as compared with Exe alone, in non-steroidal AI (NSAI)-pretreated post-menopausal patients affected by HR+/HER2-MBC, while maintaining health-related quality of life (HRQoL) (9-11).

Over the past two years, a new class of drugs, namely, CDK4/6 inhibitors, in combination with letrozole or fulvestrant as first or second-line therapy, respectively, has increasingly been used in the treatment of luminal MBC disease. Despite the impressive results from recently published studies reporting a significant PFS benefit with CDK4-6 inhibitors (palbociclib, ribociclib, abemaciclib) in combination with ET compared with ET alone, overall survival (OS) data are still awaited and the optimal sequence of ET, as monotherapy or in combination with targeted agents, is still not well established (12-15). However, combination of CDK4-6 inhibitors and letrozole is reasonably expected to become the new gold standard as first-line therapy in post-menopausal patients with HR+/HER2-endocrine sensitive MBC.

Nevertheless, it is crucial to acquire data about long-term outcomes and toxicity profile of therapeutic options currently used in everyday clinical practice in order to define the best therapeutic strategy in HR+/HER2-advanced breast cancer (ABC). Of note, the ongoing phase III randomized trial GIM-16 FEVEX (EudraCT n. 2014-004035-38), where MBCs have been randomized to receive Exe-Eve, followed, in case of progression disease, by fulvestrant or vice versa, should define the best therapeutic sequence in second and third line.

In our multicenter observational study, we retrospectively analyzed data from $264 \mathrm{HR}+$ /HER2- MBC patients who received Exe-Eve combination, following NSAI failure, in different lines of hormonal treatment to evaluate the efficacy and tolerability of this combination in the 'real world' setting.

\section{Patients and methods}

The main aim of our retrospective study was the analysis of activity, efficacy, and safety of Exe-Eve treatment according to the line of therapy. From January 2012 to January 2017, 264 patients with HR+/HER-MBC who received Exe-Eve as first or further line of ET, were eligible for the final analysis. For all patients, inclusion criteria were histologically confirmed diagnosis of HR+ and HER-locally advanced or MBC, age $\geq 18$ years, Eastern Cooperative Oncology Group (ECOG) performance status (PS) $\leq 2$, basal screening for hepatitis $\mathrm{B}$ and $\mathrm{C}$, administration of at least one cycle of Exe-Eve until disease progression, unacceptable toxicity or patient refusal, availability of clinical-pathological, radiologic, and laboratory parameters before Exe-Eve treatment, response evaluation, and survival data.

Furthermore, prior therapy with AI was permitted if recurrence had occurred during or within 12 months of completion of adjuvant therapy, or in case of progression during treatment for advanced stage disease; likewise, previous chemotherapy for metastatic disease and palliative radiotherapy on bone and/or brain were permitted. Eve starting dose was $10 \mathrm{mg}$ once a day orally; a dose reduction $(5 \mathrm{mg}$ ) was chosen by the caring physician in some instances. Supportive measures were allowed and implemented according to individual daily practice. Toxicity data were collected monthly at each patient visit and were classified according to the National Cancer Institute Common Criteria for Adverse Events (NCI-CTCAE), version 4 (16).

Accordingly, in case of toxicity, dose adjustments or temporary interruptions of therapy as well as timing and modality of evaluation were independently implemented by each investigator (as a general rule, Eve dose was reduced for toxicity of grade 2 or higher, while temporary interruption of treatment was proposed for mild toxicities; tumor assessment was carried out every 3 months). Data were retrieved after an anonymization procedure from a centralized database at the Breast Unit of Cardarelli Hospital. Four patients were lost to follow-up, and the study was completed by January 31, 2017. All patients provided written informed consent about the use of their data for future medical research. The Institutional Review Board at 'F. Magrassi' Department of Clinical and Experimental Medicine of 'Luigi Vanvitelli' University of Campania, Naples (Italy) approved the study.

Statistical analysis. All continuous data were expressed as mean $\pm \mathrm{SD}$, range and median value; frequencies and percentages were reported for categorical variables. Fisher's exact test was used to analyze associations with categorical variables. Survival distribution was estimated by the Kaplan-Meier method with 95\% confidence interval(CI) (17). Progression Free Survival (PFS) was defined as the time elapsed between the first Exe-Eve dose to the detection of disease progression or death for any cause. Patients who died of causes other than breast cancer-without experiencing tumor progression-were regarded as censored events at the date of death when computing the PFS rate. Differences in PFS according to clinical parameters or line of treatment were evaluated by the log-rank test and described by the Kaplan-Meier method. For final analysis, the PFS status of all patients was updated within 1 month of January 2017 deadline. Overall survival (OS) was defined as 
the time from the first cycle of therapy with Exe-Eve to the date of death or last contact. Cox proportional-hazards model was applied to multivariate survival analysis, and P-values and hazard ratios (HRs) with 95\% CI were obtained. All significant variables in the univariate model were used to build the multivariate model of survival. Statistical Package for the Social Sciences (SPSS) 20.0 software, (Chicago, IL, USA) was used for statistical analysis and integrated with Medcalc software V.9.4.2.0 (Mariakerke, Belgium). In all analyses, the significance level was specified as $\mathrm{P}<0.05$.

\section{Results}

Efficacy. Exe-Eve combination was used as first, second, or third line of ET in 45 (17\%), 115 (43.6\%), and 104 (39.4\%) patients with MBC (total: 264; median age 56 years, range 49-64), respectively. Of these, 192 (72.7\%) and 229 (86.7\%) received adjuvant chemotherapy and ET, respectively. Furthermore, 128 patients (48.5\%) treated with chemotherapy as first line treatment for metastatic disease subsequently received Exe-Eve combination as first (3 patients), second (56 patients), or third (69 patients) hormonal line. The main characteristics of the series are reported in Table I. At the end of the study, 104 patients (39.4\%) had died and 156 patients $(59.1 \%)$ were still alive, while $4(1.5 \%)$ patients were lost to follow-up; thus, 260 patients $(43,114$, and 103 in first, second, or third line of treatment, respectively) were eventually considered for survival analysis.

Exe-Eve combination was shown to be active in all lines of therapy. Particularly, although no complete response was observed, 105 (39.8\%) partial responses, 88 (33.3\%) disease stabilizations, and $71(26.9 \%)$ disease progressions were recorded. The overall Disease Control Rate (DCR) was $73.1 \%$, with no statistically significant difference between the different settings $(73.3,79.2$ and $66.3 \%$ in first, second and third-line therapy, respectively; $\mathrm{P}=0.105$ ).

At the time of data censoring, $4.1 \%$ of patients were receiving Exe-Eve without evidence of disease progression; median PFS was 9.1 months (95\% CI 7.4-10.2) (Fig. 1). At a median follow-up of 42 months, median PFS was $11.6(95 \% \mathrm{CI}$ 5.8-17.3), 9.7 (95\% CI7.7-10.8), and 7.5 (95\% CI 6.0-9.8) months for patients treated with Exe-Eve as first, second, or third line of treatment, respectively, with a statistically significant correlation on univariate analysis with younger age $(\mathrm{P}=0.024)$, no previous adjuvant chemotherapy $(\mathrm{P}=0.028)$, no previous adjuvant ET $(\mathrm{P}=0.030)$, adjuvant $\mathrm{ET}$ duration $\geq 36$ months $(\mathrm{P}=0.013)$, involvement of liver and/or lung $(\mathrm{P}=0.054)$, no previous chemotherapy for metastatic disease $(\mathrm{P}=0.0050)$, $\mathrm{PS}=0$ at the start of treatment $(\mathrm{P}<0.0001)$. The risk of disease progression between the different subgroups was not statistically significant (log-rank P-value=0.218) (Fig. 2).

On multivariate analysis, previous adjuvant ET $(\mathrm{P}=0.001$; $\mathrm{HR}=2.78$; 95\% CI: 1.68-4.60), previous chemotherapy for advanced disease $(\mathrm{P}=0.0027 ; \mathrm{HR}=1.39 ; 95 \% \mathrm{CI}: 1.03-1.89)$, and poor $\mathrm{PS}(\mathrm{P}=0.002 ; \mathrm{HR}=2.70 ; 95 \% \mathrm{CI}$ : 1.67-4.37) were shown to be independent prognostic factors related to poor recurrence rate. Conversely, the risk of progression was significantly lower in patients treated with hormonal drugs for 36 months or longer $(\mathrm{HR}=0.48$; 95\% CI: $0.34-0.68)$ in the adjuvant setting.
Median OS was 33.0 months (95\% CI 25.2-41.2) (Fig. 3); at a median follow-up of 67 months, median OS was 43.1 months (95\% CI 23.1-53.2), 31.7 months (95\% CI 23.5-44.1) and 27.9 months (95\% CI 22.7-38.9) for patients treated with Exe-Eve as first, second, or third line of treatment, respectively (the difference between the different OS medians was not statistically significant, $\mathrm{P}=0.538$ ) (Fig. 4). On univariate analysis, the variables related to better survival were younger age $(\leq 48$ years) $(\mathrm{P}=0.041), \mathrm{Ki} 67 \leq 20 \%(\mathrm{P}=0.032)$, duration of adjuvant $\mathrm{ET} \geq 36$ months $(\mathrm{P}=0.028)$, disease-free interval $\geq 45$ months $(\mathrm{P}=0.010)$, no previous chemotherapy for metastatic disease $(\mathrm{P}=0.001)$, ECOG $\mathrm{PS}=0(\mathrm{P}=0.032)$, duration of treatment with Exe-Eve longer than 6.3 months $(\mathrm{P}=0.012)$, partial response $(\mathrm{P}=0.009)$, presence of stomatitis $(\mathrm{P}=0.049)$, and absence of diabetes during the treatment $(\mathrm{P}=0.001)$. Finally, on multivariate analysis, the variables related to a worse outcome were diabetes $[\mathrm{P}=0.001 ; \mathrm{HR}=2.35 ; 95 \% \mathrm{CI}$ 1.52-3.58)] and previous chemotherapy for metastatic disease $(\mathrm{P}=0.032$; HR=1.58; 95\% CI 0.92-2.26); conversely, the presence of mucositis correlated with long-term survival $(\mathrm{P}=0.042$; $\mathrm{HR}=0.64 ; 95 \%$ CI $0.30-0.82$ ).

Toxicity. Overall, Exe-Eve was fairly well tolerated, most toxicities being G1 or 2, while treatment discontinuation was required in 15 patients $(5.7 \%)$ due to unacceptable toxicity. Particularly, one patient who received Exe-Eve as first line treatment suffered from G4 diarrhea and refused the treatment, 3 patients developed interstitial lung disease requiring hospitalization, and 1 case of persistent G4 neutropenia was registered among second line patients. Finally, in patients subjected to a third line of therapy, 3 cases of G3 pneumonitis, 3 cases of G4 diarrhea, 1 case of persistently increased hepatic transaminases, 1 case of hyperglycemia requiring insulin therapy and a brief hospitalization, and 2 cases of G4 stomatitis were recorded.

Two hundred and fifty patients were started on full dose Eve, while 16 patients $(6 \%)$ began treatment with half dose per physician choice; due to toxicities, Eve dosage was reduced to $5 \mathrm{mg}$ in 25 patients $(9.45 \%)$.

Overall, hematologic toxicity was mild and no G4 events were recorded, with the exception of the neutropenic patient described above. Of note, a significant difference among the three groups of patients was recorded in terms of incidence of G1-3 thrombocytopenia and neutropenia, which occurred more frequently in second and third line, respectively (Table II).

The most commonly reported extra-hematologic toxicities (all grades) were stomatitis (54.8\%), fatigue (39.3\%), rash (33.3\%), and diarrhea (33.2\%), which occurred in more than one third of patients (Table II). Nausea and decreased appetite were recorded in 28.7 and $27.6 \%$ of patients, respectively, even if G3-4 adverse events (AEs) were rare (about 1-2\%). Elevated serum transaminases, cough, hyperglycemia, pneumonitis/interstitial lung disease were also observed and the majority of G3-4 toxicities occurred in patients treated with Exe-Eve as third line therapy.

\section{Discussion}

The outcome of MBC patients is continuously improving because of the availability of new active therapeutic options. 
Table I. Patient characteristics.

\begin{tabular}{|c|c|c|c|c|c|}
\hline Characteristic & $\begin{array}{c}\text { Exe-Eve as I } \\
\text { line of ET }(\%)\end{array}$ & $\begin{array}{l}\text { Exe-Eve as II } \\
\text { line of ET }(\%)\end{array}$ & $\begin{array}{l}\text { Exe-Eve as III } \\
\text { line of ET }(\%)\end{array}$ & Total $(\%)$ & P-value \\
\hline Total & 45 & 115 & 104 & 264 & \\
\hline Age (years) & & & & & 0.091 \\
\hline$\leq 48$ & $6(13.3)$ & $31(27.0)$ & $28(26.9)$ & $65(24.6)$ & \\
\hline $49-64$ & $25(53.3)$ & $65(56.5)$ & $58(55.8)$ & $147(55.7)$ & \\
\hline$\geq 65$ & $15(33.3)$ & $19(16.5)$ & $18(17.3)$ & $52(19.7)$ & \\
\hline ECOG PS & & & & & 0.724 \\
\hline 0 & $37(82.2)$ & $100(87.0)$ & $90(86.5)$ & $227(86)$ & \\
\hline $1-2$ & $8(17.8)$ & $15(13.0)$ & $14(13.5)$ & $37(14.0)$ & \\
\hline Surgery (primary tumor) & & & & & 0.082 \\
\hline Quadrantectomy & $28(62.2)$ & $56(48.7)$ & $44(42.3)$ & $128(48.5)$ & \\
\hline Mastectomy/biopsy & $17(37.8)$ & $59(51.3)$ & $60(57.7)$ & $136(51.5)$ & \\
\hline $\mathrm{pT}$ & & & & & 0.082 \\
\hline 1.2 & $17(37.8)$ & $91(79.1)$ & $72(69.2)$ & $201(76.1)$ & \\
\hline 3.4.x & $7(15.6)$ & $24(20.9)$ & $32(30.8)$ & $63(23.9)$ & \\
\hline $\mathrm{pN}$ & & & & & 0.211 \\
\hline 0 & $18(40.0)$ & $24(20.8)$ & $18(17.3)$ & $60(22.7)$ & \\
\hline 1 & $10(22.2)$ & $26(22.6)$ & $26(25.0)$ & $62(23.5)$ & \\
\hline 2 & $8(17.8)$ & $34(29.5)$ & $29(27.8)$ & $71(26.9)$ & \\
\hline 3 & $5(11.1)$ & $21(18.2)$ & $20(19.2)$ & $46(17.4)$ & \\
\hline $\mathrm{X}$ & $4(8.8)$ & $10(8.6)$ & $11(6.7)$ & $25(9.5)$ & \\
\hline Grading & & & & & 0.510 \\
\hline G1-G2 & $17(37.8)$ & $52(45.2)$ & $50(48.1)$ & $119(45.1)$ & \\
\hline G3 & $28(62.2)$ & $63(54.8)$ & $54(51.9)$ & $145(54.9)$ & \\
\hline ER, median (IQR) & $80(70-90)$ & $80(70-90)$ & $80(60-90)$ & $80(70-90)$ & 0.252 \\
\hline PgR, median (IQR) & $60(30-80)$ & $60(30-80)$ & $60(20-80)$ & $60(30-80)$ & 0.804 \\
\hline KI67 & & & & & 0.852 \\
\hline$\leq 20 \%$ & $22(48.9)$ & $56(48.7)$ & $47(45.2)$ & $125(47.4)$ & \\
\hline$>20 \%$ & $23(51.1)$ & $59(51.3)$ & $57(54.8)$ & $139(52.7)$ & \\
\hline Adjuvant chemotherapy & & & & & 0.641 \\
\hline Yes & $35(77.8)$ & $81(70.4)$ & $76(73.1)$ & $192(72.7)$ & \\
\hline No & $10(22.2)$ & $34(29.6)$ & $28(26.9)$ & $72(27.3)$ & \\
\hline Adjuvant radiotherapy & & & & & 0.002 \\
\hline Yes & $38(84.4)$ & $64(60)$ & $56(53.9)$ & $163(61.7)$ & \\
\hline No & $7(15.6)$ & $46(40)$ & $48(46.2)$ & $101(38.3)$ & \\
\hline Adjuvant ET & & & & & 0.015 \\
\hline Yes & $45(100)$ & $96(83.5)$ & $88(84.6)$ & $229(86.7)$ & \\
\hline No & $0(0)$ & $19(16.5)$ & $16(15.4)$ & $35(13.3)$ & \\
\hline Median duration of adjuvant ET, months (IQR) & $42(21-60)$ & $38(12-60)$ & $35.5(16-60)$ & $36(15-60)$ & 0.711 \\
\hline Disease free interval (from surgery) & $42(25-93)$ & $44(18-84)$ & $48.5(22-84)$ & $45(21-85)$ & 0.710 \\
\hline 1st site of metastases & & & & & 0.005 \\
\hline Liver/lung & $11(24.4)$ & $13(11.3)$ & $10(9.6)$ & $34(12.9)$ & \\
\hline Skin/lymph nodes/peritoneo/pleura & $4(8.9)$ & $21(18.3)$ & $32(30.8)$ & $57(21.6)$ & \\
\hline Bone & $30(66.7)$ & $81(70.4)$ & $62(59.6)$ & $173(65.5)$ & \\
\hline 2 site of metastases & & & & & 0.406 \\
\hline No & $20(44.4)$ & $61(53.0)$ & $56(53.9)$ & 137 (51.9) & \\
\hline Liver, lung & $13(28.9)$ & $20(17.4)$ & $16(15.4)$ & $49(18.6)$ & \\
\hline Other & $12(26.7)$ & $34(29.6)$ & $32(30.8)$ & $78(29.6)$ & \\
\hline
\end{tabular}


Table I. Continued.

\begin{tabular}{|c|c|c|c|c|c|}
\hline Characteristic & $\begin{array}{c}\text { Exe-Eve as I } \\
\text { line of ET }(\%)\end{array}$ & $\begin{array}{l}\text { Exe-Eve as II } \\
\text { line of ET (\%) }\end{array}$ & $\begin{array}{l}\text { Exe-Eve as III } \\
\text { line of ET }(\%)\end{array}$ & Total $(\%)$ & P-value \\
\hline 3 site of metastases & & & & & 0.999 \\
\hline No & $41(91.1)$ & $105(91.3)$ & $95(91.4)$ & $241(91.3)$ & \\
\hline Yes & $4(8.9)$ & $10(8.7)$ & $9(8.7)$ & $23(8.7)$ & \\
\hline 1st line CT for metastatic disease & & & & & $<0.001$ \\
\hline Yes & $3(6.7)$ & $56(48.7)$ & $69(66.4)$ & $128(48.5)$ & \\
\hline No & $42(93.3)$ & $59(51.3)$ & $35(33.7)$ & $136(51.5)$ & \\
\hline
\end{tabular}

ER, estrogen receptor; PgR, progesterone receptor; CT, computed tomography; ET, endocrine therapy; PS, performance status; IQR, interquartile range.

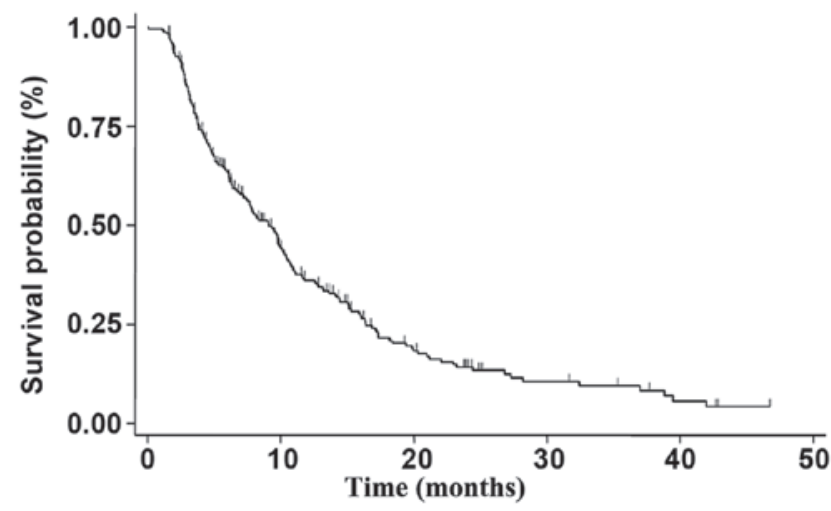

Figure 1. mPFS in the overall population. The mPFS in the overall population treated with Everolimus-Exemestane in combination was 9.1 months (95\% confidence interval, 7.4-10.2). mPFS, median progression-free survival.

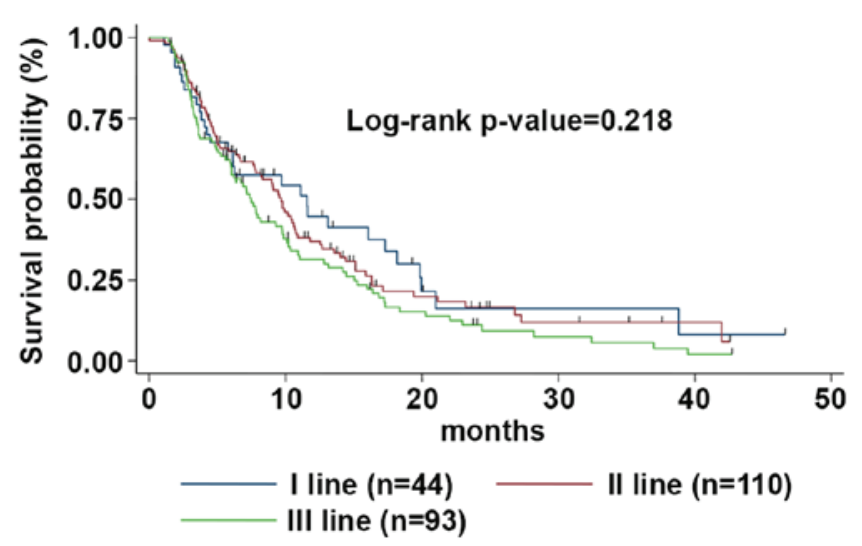

Figure 2. mPFS according to the line of treatment. The mPFS was 11.6 (95\% CI, 5.8-17.3), 9.7 (95\% CI, 7.7-10.8) and 7.5 (95\% CI, 6.0-9.8) months for patients treated with Exe-Eve as first, second or third line of treatment, respectively. mPFS, median progression-free survival; Eve-Exe, Everolimus-Exemestane; CI, confidence interval.

The challenge for clinicians is to balance treatment-related toxicity with the likelihood of benefit and cancer-related symptom relief deriving from therapy. For this reason, current clinical guidelines advocate the use of ET as the preferred treatment for HR+, HER2- MBC, unless visceral crisis or concern/proof of endocrine resistance is observed.

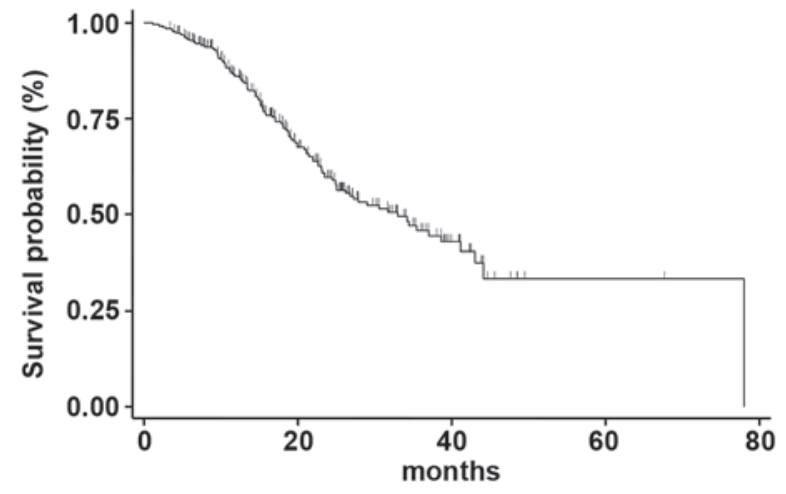

Figure 3. mOS in the overall population. The mOS in the overall population treated with Everolimus-Exemestane in combination was 33.0 months (95\% confidence interval 25.2-41.2) mOS, median overall survival.

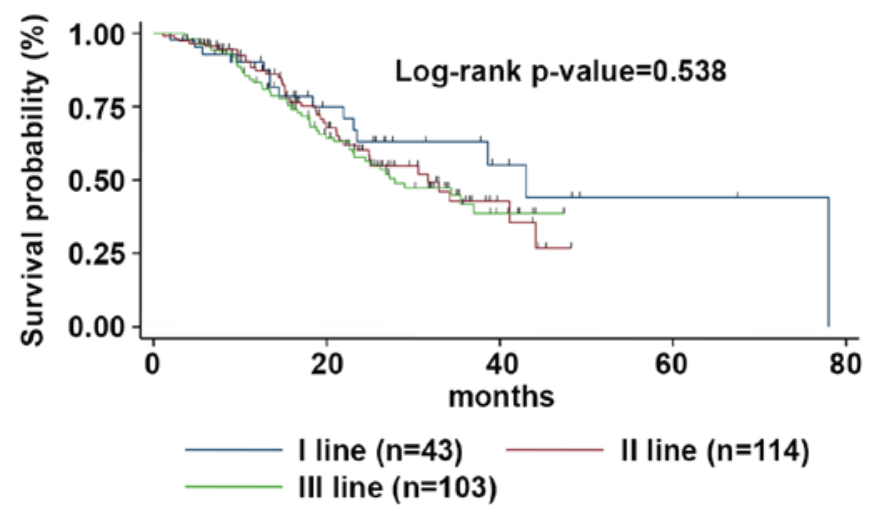

Figure 4. mOS according to the line of treatment. The mOS was 43.1 (95\% CI, 23.1-53.2), 31.7 (95\% CI, 23.5-44.1), and 27.9 months (95\% CI, 22.7-38.9) for patients treated with Exe-Eve at first, second or third line of treatment, respectively. mOS, median overall survival; Eve-Exe, Everolimus-Exemestane; CI, confidence interval.

Recently, the introduction of CDK4/6 inhibitors in combination with endocrine agents resulted in a significant PFS benefit, however, overall survival data are still missing and there is no consensus on the optimal sequence of ET, that is, as monotherapy or in combination with targeted agents (12-15). 
Table II. Haematological and non-haematological toxicities.

\begin{tabular}{|c|c|c|c|c|c|}
\hline Haematological toxicities & All patients (\%) & First line $(\%)$ & Second line $(\%)$ & Third line $(\%)$ & P-value ${ }^{a}$ \\
\hline Neutropenia & & & & & 0.002 \\
\hline G1-2 & $36(13.6)$ & $2(4.4)$ & $12(10.4)$ & $22(21.1)$ & \\
\hline G3-4 & $6(2.2)$ & - & $2(1.7)$ & $4(3.8)$ & \\
\hline Anemia & & & & & 0.788 \\
\hline G1-2 & $65(24.6)$ & $13(28.9)$ & $28(24.3)$ & $24(23.0)$ & \\
\hline G3-G4 & $13(4.9)$ & $2(4.4)$ & $4(3.5)$ & $7(6.7)$ & \\
\hline Thrombocytopenia & & & & & 0.010 \\
\hline G1-2 & $32(12.1)$ & - & $18(15.6)$ & $14(13.5)$ & \\
\hline G3-4 & $6(2.28)$ & - & $3(2.6)$ & $3(2.9)$ & \\
\hline \multicolumn{6}{|l|}{ Non-haematological toxicities } \\
\hline Stomatitis & & & & & 0.828 \\
\hline G1-2 & $120(45.4)$ & $15(33.3)$ & $55(47.8)$ & $50(48.0)$ & \\
\hline G3-4 & $25(9.5)$ & $4(8.9)$ & $10(8.7)$ & $11(10.6)$ & \\
\hline Fatigue & & & & & 0.279 \\
\hline G1-2 & $95(36.0)$ & $15(33.3)$ & $39(34.0)$ & $41(39.4)$ & \\
\hline G3-4 & $9(3.4)$ & - & $3(2.6)$ & $6(5.8)$ & \\
\hline Rash & & & & & 0.778 \\
\hline G1-2 & $84(31.8)$ & $16(35)$ & $36(31.3)$ & $32(30.8)$ & \\
\hline G3-G4 & $4(1.5)$ & $1(2.2)$ & $1(<0.1)$ & $2(1.9)$ & \\
\hline Diarrhea & & & & & 0.876 \\
\hline G1-2 & $76(28.8)$ & $15(33.3)$ & $29(25.2)$ & $32(30.7)$ & \\
\hline G3-4 & $12(4.5)$ & $2(4.0)$ & $4(3.5)$ & $6(5.7)$ & \\
\hline Nausea & & & & & 0.627 \\
\hline G1-2 & $76(28.7)$ & $12(26.7)$ & $32(27.8)$ & $32(30.8)$ & \\
\hline G3-G4 & $3(1.1)$ & - & $1(<0.1)$ & $2(1.9)$ & \\
\hline Decreased appetite & & & & & 0.727 \\
\hline G1-2 & $73(27.6)$ & $10(22.2)$ & $28(24.3)$ & $35(33.6)$ & \\
\hline G3-G4 & $3(1.1)$ & - & $1(<0.1)$ & $2(1.9)$ & \\
\hline AST increase & & & & & 0.781 \\
\hline G1-2 & $28(10.6)$ & $5(11.1)$ & $11(9.6)$ & $12(11.5)$ & \\
\hline G3-4 & $9(3.4)$ & $1(2.2)$ & $3(2.6)$ & $5(4.8)$ & \\
\hline Cough & & & & & 0.192 \\
\hline G1-2 & $31(11.7)$ & $5(11.1)$ & $12(10.4)$ & $14(13.5)$ & \\
\hline G3-G4 & $3(1.1)$ & - & - & $3(2.9)$ & \\
\hline ALT increase & & & & & 0.375 \\
\hline G1-2 & $24(9.1)$ & $5(11.1)$ & $10(8.7)$ & $9(8.6)$ & \\
\hline G3-4 & $7(2.6)$ & - & $3(2.6)$ & $4(3.8)$ & \\
\hline Hyperglicemia & & & & & 0.472 \\
\hline G1-2 & $17(6.4)$ & $3(6.7)$ & $8(6.9)$ & $6(5.8)$ & \\
\hline G3-4 & $5(1.8)$ & - & $2(1.7)$ & $3(2.9)$ & \\
\hline Pneumonitis/interstitial lung disease & & & & & 0.592 \\
\hline G1-2 & $13(4.9)$ & $2(4.4)$ & $4(3.5)$ & $7(6.7)$ & \\
\hline G3-G4 & $6(2.3)$ & - & $2(1.7)$ & $4(3.8)$ & \\
\hline
\end{tabular}

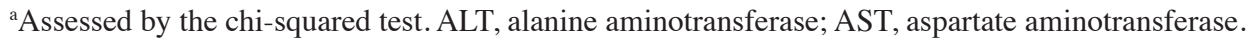

Currently, the combination of Eve and Exe is a widely used regimen for treatment of endocrine sensitive postmenopausal
MBC patients progressing after NSAIs therapy. However, current international guidelines suggest taking into account 
the relevant class-effect AEs of this regimen with regard to the decision to treat, which nonetheless should be made on a case by case basis.

For this purpose, more experience has to be collected on toxicity profile and efficacy in different subsets of patients (i.e., differing in metastatic sites, performance status, number of prior therapies etc.) in order to help physicians to optimize the therapeutic strategy for each patient.

The main aim of our observational study was to explore the efficacy and tolerability of Eve-Exe combination, according to the line of therapy, in an unselected population from nine Cancer Centers in Campania region (South Italy). The hypothesis of the association between the onset of side effects and efficacy was also addressed in our analysis.

The pivotal phase III BOLERO-2 trial showed that dual-blockade based on the association of Eve plus Exe doubled the median PFS compared to Exe alone (7.8 vs. 3.2 by investigator review and 11 vs. 4.1 months by central review, respectively) in patients with $\mathrm{HR}+$, HER2-MBC progressing after prior NSAIs. The clinical benefit rate (CBR), defined as $\mathrm{CR}+\mathrm{PR}+\mathrm{SD} \geq 24$ months, was also better in the Eve-Exe arm with respect to Exe alone arm (51.3\% vs. $26.4 \%$, respectively).

OS was a secondary endpoint but did not meet statistical significance despite it was shown to increase (4.4 months) following addition of Eve to Exe (31.0 months in the experimental arm vs. 26.6 months in patients receiving Exe alone) $(9-10,18)$.

The lack of a statistically significant survival gain could be due to the fact that BOLERO-2 trial was powered to detect only an eight-month OS improvement. Furthermore, the small imbalance in post-study salvage chemotherapy use (63 vs. $53 \%$, control vs. experimental arm, respectively) has probably influenced OS results leading to a reduction in the survival gap between the two groups.

In our retrospective analysis, Exe-Eve combination was shown to be active in all lines of therapy. Particularly, although no complete response was observed, DCR was $73.1 \%$, with no statistically significant difference between the different settings. At a median follow-up of 42 months, median PFS was 11.6, 9.7, and 7.5 months for patients treated with Exe-Eve as first, second, or third line of treatment, respectively. Median OS was 33.0 months; at a median follow-up of 67 months, median OS was 43.1, 31.7, and 27.9 months for patients treated with Exe-Eve as first, second or third line of treatment, respectively. No statistically significant difference in terms of PFS and OS between the different subgroups was detected. Our results confirmed the considerable activity and efficacy of Eve-Exe observed in BOLERO-2, although the safety profile of this combination remains debated. In the BOLERO-2 trial, the most commonly reported AEs, affecting at least one-third of patients in the Eve-Exe arm, were stomatitis, rash, fatigue, diarrhea, nausea, decreased appetite, and pneumonitis. While most AEs were low grade (G1-2), one-half of patients in the Eve-Exe arm experienced grade 3-4 toxicities vs. $27 \%$ of patients in the placebo plus Exe arm. The discontinuation rate due to AEs was higher with Eve-Exe (9\%) compared with the control arm (3\%); the most common toxiticies leading to treatment discontinuation in the experimental group included pneumonitis, stomatitis, dyspnea, and fatigue (19). Consistent with BOLERO-2 results, in our analysis, the most frequently described toxicities (all grades) were stomatitis (54.8\%), fatigue (39.3\%), rash $(33.3 \%)$, and diarrhea $(33.2 \%)$, which occurred in more than one third of patients. Treatment discontinuation was registered only in $5.7 \%$ of patients and the most common AEs leading to treatment discontinuation were pneumonitis, stomatitis, and diarrhea. In the Italian expanded-access, multicenter BALLET trial, permanent treatment discontinuation due to side effects was reported to be more frequent $(17.1 \%$ of patients) with respect to our series and was mainly caused by non infectious pneumonitis (NIP), stomatitis, asthenia, and dyspnea. The majority of these toxicities occurred within the first 12 weeks of start of therapy, consistently across all clinical trials (20). Since 2012 approval, several retrospective trials have been carried out to assess the role of Eve-Exe combination in daily clinical practice, providing clinicians with valuable additional data to guide treatment decisions. To our knowledge, five large observational studies have strengthened the role of this combination in patients with HR+, HER2-MBC. With the exception of the Italian trial by Moscetti et al (21), the remaining studies were only presented as abstracts at scientific international conferences on breast cancer.

The Austrian non-interventional phase 4 STEPAUT study aimed at evaluating efficacy and safety of Eve-Exe according to clinical routine. The second interim analysis on 225 out of 300 enrolled patients was recently presented at San Antonio Breast Cancer Symposium in 2016. Overall, median PFS was 9.5 months, in line with our PFS data (9.1 months). A subgroup analysis was also performed according to Eve dosing and, interestingly, PFS was lower for the $5 \mathrm{mg}$-group compared to the 10-mg group (6.5 vs. 9.1 months, respectively). However, it should be noted that patients receiving the $5 \mathrm{mg}$ starting dose had unfavorable prognostic factors as well as worse ECOG PS and more prior therapies. The safety profile was consistent with data previously reported in BOLERO-2 trial and, noteworthy, in accordance with our findings, occurrence of stomatitis did not negatively affect PFS. The EVEREXES phase IIIb trial investigated safety and tolerability profiles as primary endpoints and efficacy as a secondary objective. In this trial, 232 post-menopausal women affected by HR+, HER2-MBC were recruited in Eastern countries. The planned interim analysis presented at San Antonio Breast Cancer Symposium by Im et al in December 2015 showed a median PFS of 9.45 months, which is also consistent with our results. The majority of AEs were grade 1-2 and the most common G3-4 toxicities, as expected, included stomatitis, fatigue, hyperglycemia, and NIP. Therefore, this trial confirmed the role of Eve-Exe combination even for the treatment of patients from Asia, Africa and middle Est, a population poorly represented in BOLERO-2 ( $<10 \%$ of all series). Furthermore, a large, multicenter, non interventional study from Germany (BRAWO trial) including 3,000 patients provided data on the routine clinical use of Eve-Exe therapy. The second interim analysis of BRAWO, presented at the European Society of Medical Oncology (ESMO) in September 2014 by Fasching et al was carried out on 500 patients: A median PFS of 8 months was recorded, with a higher median PFS (10.1 months) for patients treated with Exe-Eve in the first-line setting. These data are still in accordance with our results as an increased PFS of 11.6 months was observed in patients receiving Eve-Exe as first line treatment, even though no statistically significant 
correlation in terms of risk of disease progression between the different lines of treatment was found. Of note, the third interim analysis of BRAWO study, presented at ESMO in 2015, showed a significantly longer duration of treatment in patients with younger age, better ECOG performance status, and no comorbidities. 4EVER is another German phase IIIb study evaluating the efficacy and safety of Eve-Exe in a broader patient population than that of BOLERO-2. The final efficacy analysis, calculated on 281 patients, was presented at the San Antonio Breast Cancer Symposium in 2015. Efficacy data were poorer compared with those from BOLERO-2, being the median PFS equal to 5.6 months. This may be due to the different patient population enrolled in this trial. In fact, unlike the BOLERO-2 trial, there were less limitations in enrollment criteria, including the number of previous CT lines, the prior use of Exe, or the time to progression after NSAI therapy. Finally, Moscetti et al (21) recently published an Italian retrospective trial assessing the safety of Eve-Exe combination and the possible association between toxicities and previous treatments in 181 unselected patients. On multivariate analysis, no association between the number of prior therapies and toxic events was found, which is in line with the real-life data of the Italian BALLETT-related cohort (20). In this study, a switch from $10 \mathrm{mg}$ Eve starting dose to $5 \mathrm{mg}$, due to AEs, was reported in $27 \%$ of patients, which is a figure quite similar to that reported in BOLERO-2 and EAP. Conversely, in our series, Eve dosage was reduced to $5 \mathrm{mg}$ in about $10 \%$ of patients only, perhaps due to the wide use of prophylactic measures and therapeutic interventions for stomatitis management.

Another important aspect to focus on is the correlation between adverse events and patient characteristics and response to treatment. In this regard, our multivariate analysis showed diabetes and previous chemotherapy for metastatic disease as the variables related to a worse outcome; conversely, the presence of mucositis correlated with long-term survival. Recently, a meta-analysis of data from seven randomized phase III trials of Eve assessing the clinical impact of stomatitis on efficacy in different solid tumors was published (22). Specifically, in BOLERO-2 study, the median PFS was 8.5 vs. 6.9 months for patients receiving Eve with or without stomatitis within 8 weeks, respectively. These results and those of the above mentioned STEPAUT trial are in accordance with our findings and suggest a careful and close monitoring of patients in the first weeks of treatment in order to better manage and prevent stomatitis. In this scenario, the SWISH trial demonstrated that a prophylactic use of a commercially available, non-expensive oral dexamethasone mouthwash resulted in a marked reduction in the incidence and severity of stomatitis in patients receiving Eve-Exe combination for treatment of HR+, HER2-MBC, when compared with data from BOLERO-2. On the basis of these findings, this measure can be considered as a new standard of oral care for $\mathrm{BC}$ patients receiving Eve and Exe therapy (23).

In conclusion, with the limitations due to the observational nature of our findings, addition of Eve to Exe is an effective therapeutic option for HR+, HER2- MBC patients after failure of NSAI therapy in the real word scenario. Patient characteristics, costs, and side effects have to be integrated in the treatment decision making process.

\section{Acknowledgements}

Not applicable.

\section{Funding}

No funding was received.

\section{Availability of data and materials}

All data generated or analyzed during this study are included in this published article.

\section{Authors' contributions}

FR and MO conceived and designed the study. GiuC, AD, CM, GiaC, RL, AF, FN, RA, OM and PI collected the data from the source. Analysis and interpretation of data was performed by FR. AD wrote the first draft for the manuscript. FC and SD performed data collection and critical revision of the article for important intellectual content. All authors approved the final manuscript.

\section{Ethics approval and consent to participate}

The Institutional Review Board at 'F. Magrassi' Department of Clinical and Experimental Medicine of 'Luigi Vanvitelli' University of Campania, Naples (Italy) approved the study. All patients provided written informed consent for the use of their data.

\section{Patient consent for publication}

Not applicable.

\section{Competing interests}

The authors declare that they have no competing interests.

\section{References}

1. Ferlay J, Soerjomataram I, Dikshit R, Eser S, Mathers C, Rebelo M, Parkin DM, Forman D and Bray F: Cancer incidence and mortality worldwide: Sources, methods and major patterns in GLOBOCAN 2012. Int J Cancer 136: E359-E386, 2015.

2. Anderson WF, Chatterjee N, Ershler WB and Brawley OW: Estrogen receptor breast cancer phenotypes in the Surveillance, Epidemiology, and End Results database. Breast Cancer Res Treat 76: 27-36, 2002.

3. Cardoso F, Costa A, Senkus E, Aapro M, André F, Barrios CH, Bergh J, Bhattacharyya G, Biganzoli L, Cardoso MJ, et al: 3rd ESO-ESMO International Consensus Guidelines for Advanced Breast Cancer (ABC 3). Ann Oncol 28: 16-33, 2017.

4. National Comprehensive Cancer Network: NCCN Clinical Practice Guidelines in Oncology Breast Cancer Version 1.2018. https://www.nccn.org/professional/physician_gls/pdf/breast.pdf. Accessed April 6, 2018.

5. Osborne CK and Schiff R: Mechanisms of endocrine resistance in breast cancer. Annu Rev Med 62: 233-247, 2011.

6. Bachelot T, Bourgier C, Cropet C, Ray-Coquard I, Ferrero JM, Freyer G, Abadie-Lacourtoisie S, Eymard JC, Debled M, Spaëth D, et al: Randomized phase II trial of everolimus in combination with tamoxifen in patients with hormone receptor-positive, human epidermal growth factor receptor 2-negative metastatic breast cancer with prior exposure to aromatase inhibitors: A GINECO study. J Clin Oncol 30: 2718-2724, 2012. 
7. Baselga J, Semiglazov V, van Dam P, Manikhas A, Bellet M, Mayordomo J, Campone M, Kubista E, Greil R, Bianchi G, et al: Phase II randomized study of neoadjuvant everolimus plus letrozole compared with placebo plus letrozole in patients with estrogen receptor-positive breast cancer. J Clin Oncol 27: 2630-2637, 2009

8. Boulay A, Rudloff J, Ye J, Zumstein-Mecker S, O'Reilly T, Evans DB, Chen S and Lane HA: Dual inhibition of mTOR and estrogen receptor signaling in vitro induces cell death in models of breast cancer. Clin Cancer Res 11: 5319-5328, 2005.

9. Baselga J, Campone M, Piccart M, Burris HA III, Rugo HS, Sahmoud T, Noguchi S, Gnant M, Pritchard KI, Lebrun F, et al: Everolimus in postmenopausal hormone-receptor-positive advanced breast cancer. N Engl J Med 366: 520-529, 2012.

10. Yardley DA, Noguchi S, Pritchard KI, Burris HA III, Baselga J, Gnant M, Hortobagyi GN, Campone M, Pistilli B, Piccart M, et al: Everolimus plus exemestane in postmenopausal patients with $\mathrm{HR}(+)$ breast cancer: BOLERO-2 final progression-free survival analysis. Adv Ther 30: 870-884, 2013.

11. Burris HA III, Lebrun F, Rugo HS, Beck JT, Piccart M, Neven P Baselga J, Petrakova K, Hortobagyi GN, Komorowski A, et al: Health-related quality of life of patients with advanced breast cancer treated with everolimus plus exemestane versus placebo plus exemestane in the phase 3, randomized, controlled, BOLERO-2 trial. Cancer 119: 1908-1915, 2013.

12. Turner NC, Ro J, André F, Loi S, Verma S, Iwata H, Harbeck N, Loibl S, Huang Bartlett C, Zhang K, et al: Palbociclib in hormone-receptor-positive advanced breast cancer. N Engl J Med 373: 209-219, 2015.

13. Cristofanilli M, Turner NC, Bondarenko I, Ro J, Im SA, Masuda N, Colleoni M, De Michele A, Loi S, Verma S, et al: Fulvestrant plus palbociclib versus fulvestrant plus placebo for treatment of hormone-receptor-positive, HER2-negative metastatic breast cancer that progressed on previous endocrine therapy (PALOMA-3): Final analysis of the multicentre, double-blind, phase 3 randomised controlled trial. Lancet Oncol 17: 425-439, 2016.

14. Hortobagyi GN, Stemmer SM, Burris HA, Yap YS, Sonke GS, Paluch-Shimon S, Campone M, Blackwell KL, André F, Winer EP, et al: Ribociclib as first-line therapy for HR-positive, advanced breast cancer. N Engl J Med 375: 1738-1748, 2016.

15. Sledge GW Jr, Toi M,Neven P, Sohn J, Inoue K, Pivot X, Burdaeva O Okera M, Masuda N, Kaufman PA, et al: MONARCH 2: Abemaciclib in combination with fulvestrant in women with HR+/HER2-advanced breast cancer who had progressed while receiving endocrine therapy. J Clin Oncol 35: 2875-2884, 2017

16. Common Terminology Criteria for Adverse Events (CTCAE), Version 4.03, June 14, 2010. US Department of Health and Human Services. National Institutes of Health National Cancer Institute. http://evs.nci.nih.gov/ftp1/CTCAE/CTCAE_4.03_2010-06-14_Quick Reference_5x7.pdf. Accessed October 8, 2017.
17. Kaplan EL and Meier P: Nonparametric estimation from incomplete observations. J Am Stat Assoc 53: 457-481, 1958.

18. Piccart M, Hortobagyi GN, Campone M, Pritchard KI, Lebrun F, Ito Y, Noguchi S, Perez A, Rugo HS, Deleu I, et al: Everolimus plus exemestane for hormone-receptor-positive, human epidermal growth factor receptor-2-negative advanced breast cancer: Overall survival results from BOLERO-2. Ann Oncol 25: 2357-2362, 2014

19. Rugo HS, Pritchard KI, Gnant M, Noguchi S, Piccart M, Hortobagyi G, Baselga J, Perez A, Geberth M, Csoszi T, et al: Incidence and time course of everolimus-related adverse events in postmenopausal women with hormone receptor-positive advanced breast cancer: Insights from BOLERO-2. Ann Oncol 25: 808-815, 2014.

20. Jerusalem G, Mariani G, Ciruelos EM, Martin M, Tjan-Heijnen VC, Neven P, Gavila JG, Michelotti A, Montemurro F, Generali D, et al: Safety of everolimus plus exemestane in patients with hormone-receptor-positive, HER2-negative locally advanced or metastatic breast cancer progressing on prior non-steroidal aromatase inhibitors: Primary results of a phase IIIb, open-label, single-arm, expanded-access multicenter trial (BALLET). Ann Oncol 27: 1719-1725, 2016

21. Moscetti L, Vici P, Gamucci T, Natoli C, Cortesi E, Marchetti P, Santini D, Giuliani R, Sperduti I, Mauri M, et al: Safety analysis, association with response and previous treatments of everolimus and exemestane in 181 metastatic breast cancer patients: A multicenter Italian experience. Breast 29: 96-101, 2016.

22. Rugo HS, Hortobagyi GN, Yao J, Pavel M, Ravaud A, Franz D, Ringeisen F, Gallo J, Rouyrre N, Anak O and Motzer R: Meta-analysis of stomatitis in clinical studies of everolimus: Incidence and relationship with efficacy. Ann Oncol 27: 519-525, 2016.

23. Rugo HS, Seneviratne L, Beck JT, Glaspy JA, Peguero JA, Pluard TJ, Dhillon N, Hwang LC, Nangia C, Mayer IA, et al: Prevention of everolimus-related stomatitis in women with hormone receptor-positive, HER2-negative metastatic breast cancer using dexamethasone mouthwash (SWISH): A single-arm, phase 2 trial. Lancet Oncol 18: 654-662, 2017.

This work is licensed under a Creative Commons Attribution-NonCommercial-NoDerivatives 4.0 International (CC BY-NC-ND 4.0) License. 